\title{
Effective Lifetime Experimental Measurement under External Magnetic Field in Transient Dynamic State Obtained from a Square Electrical Excitation by Open Circuit Voltage Decay Method
}

\author{
Nazé Yacouba Traoré1, Raguilignaba Sam ${ }^{1,2}$, Alain Diasso², Bernard Zouma ${ }^{3}$, \\ François Zougmoré ${ }^{2}$ \\ ${ }^{1}$ University of Nazi Boni, Department of physic, Units of Sciences and Technology, Bobo Dioulasso, Burkina Faso \\ ${ }^{2}$ University of Joseph Ki-Zerbo, Department of Physic, Laboratory of Materials and Environment, Units of Sciences and \\ Technology, Ouagadougou, Burkina Faso \\ ${ }^{3}$ University of Ouagadougou, Department of Physic, Laboratory of Thermal and Renewable Energy, Units of Sciences and \\ Technology, Ouagadougou, Burkina Faso \\ Email: alinodiass@yahoo.fr
}

How to cite this paper: Traoré, N.Y., Sam, R., Diasso, A., Zouma, B. and Zougmoré, F. (2020) Effective Lifetime Experimental Measurement under External Magnetic Field in Transient Dynamic State Obtained from a Square Electrical Excitation by Open Circuit Voltage Decay Method. Energy and Power Engineering, 12, 709-715.

https://doi.org/10.4236/epe.2020.1212041

Received: October 31, 2020

Accepted: December 28, 2020

Published: December 31, 2020

Copyright $\odot 2020$ by author(s) and Scientific Research Publishing Inc. This work is licensed under the Creative Commons Attribution International License (CC BY 4.0).

http://creativecommons.org/licenses/by/4.0/ (c) (i) Open Access

\begin{abstract}
In this work, we present an experimental transient 3-Dimensionnal study for the minority charge carriers' effective lifetime measurement under magnetic field in transient dynamic state. The magnitude of the magnetic field B is varied from $0 \mathrm{mT}$ to $0.03 \mathrm{mT}$. The method used is mainly based on the open circuit voltage decay method. The solar cell is injected by a low electrical excitation which protects against capacitance effects. Our approach is based on the open circuit voltage decay response analysis. From an experimental set-up, we get the transient voltage data on a digital scope. The data are used for plotting transient voltage decay curves. The curves obtained and analyzed are fitted in their linear zone. This zone presents an ideal decay which permits to get good values of lifetime. The slope of the linear decay is inversely proportional to effective lifetime. The results of fitting permit determinate the effective charge carriers' lifetime directly. The results obtained are then presented and analyzed. The observations indicate that the charge carriers effective lifetime decrease when the magnetic field increases.
\end{abstract}

\section{Keywords}

Effective Lifetime, Electrical Excitation, Frequential Dynamic Regime, 
Magnetic Field

\section{Introduction}

High lifetimes are indicative of the best efficiency of solar cell while low lifetimes might point problems that reduce the quality of solar cell. In the last years, a number of new methods have been proposed and earlier methods reviewed for the measurement of minority carrier lifetime in solar cell. These include the open circuit voltage, reverse recovery, photoconductivity decay, short circuit capacitance measurement, spectral response, impedance measurement, etc.

The lifetime measurement is therefore used to assess the solar cell efficiency. For this purpose, several technics are developed by authors for lifetime measurement and their limits are pointed out [1] according to the solar cell operating mode. Among these technics, the open circuit voltage decay technic [2] [3] is a simple non-destructive method for measuring minority carriers' lifetime in solar cell. In practice, this technic tends to be subjective and unreliable because the difficulty frequently arises in finding a region of the decay which reasonably linear. However, there are been recent developments in both experimental [4] and theoretical [5] areas which are significantly improved the reliability and accuracy of the approach. In this method, a voltage is induced in the solar cell and is then allowed to decay under open circuit conditions.

In this experimental study, in first time, we present the experimental set-up. In second time, the experimental conditions will be described and assumptions made will be presented. Thirdly, the method for charge carriers' effective lifetime measurement will be explained and the results obtained will be presented and analyzed.

\section{Materials and Methods}

The experimental set-up is presented on Figure 1. The experimental set-up is composed by the following details: a mono-facial silicon solar cell manufactured by MOTCH INDUSTRY; a pulsed light source MINISTROB PHIWE, a digital oscilloscope TECKTRONIX model TDS 210, a computer INTEL 586, a low frequency generator (GBF) GFG-8015 G and Helmholtz Coil.

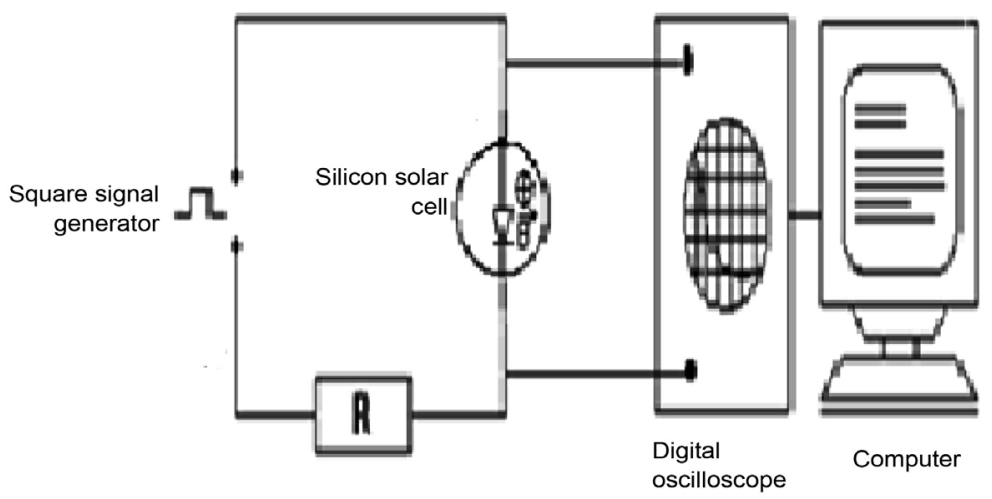

Figure 1. Experimental set-up. 


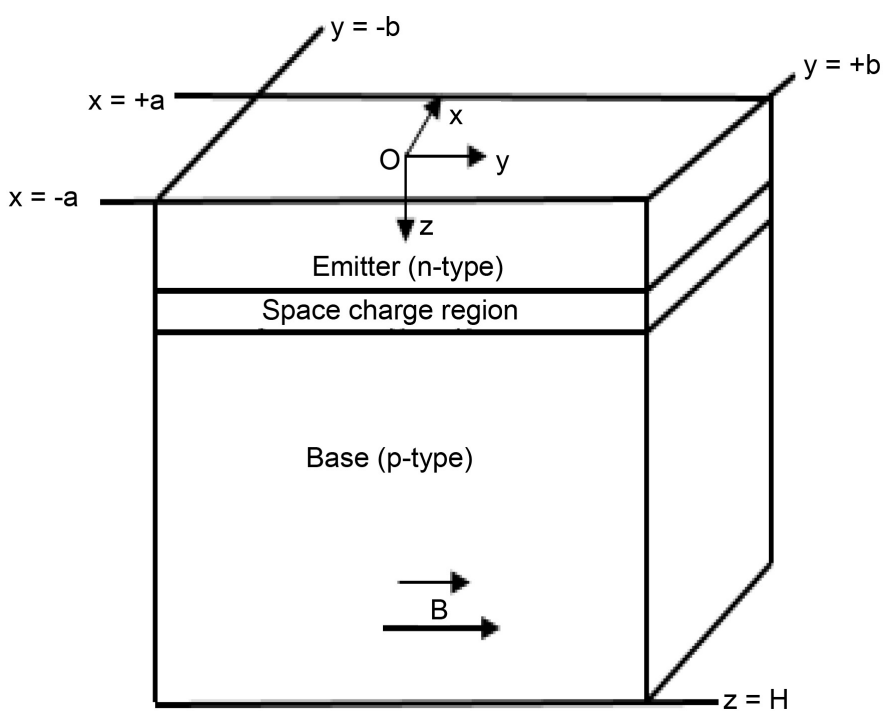

Figure 2. A sample grain under magnetic field.

At time $t=0$, the solar cell is injected by an electrical excitation which established a steady state characterize by the potential V1 corresponding to an operating point called point 1 . The voltage V1 drops from V1 to V2 (Figure 3).

At the time $\mathrm{t}=\mathrm{Te}$, the flash is abruptly stopped. The voltage V1 drops from V1 to $\mathrm{V} 2$ corresponding to a new operating point denoted 2 . The decay of voltage from V1 to V2 is recorded on a digital scope connected to a computer via RS232. The computer permits to store the experimental data for later using.

The experimental system principle operating is the same described by Sam et al. and R. Sam et al. [6] [7]. In addition, we consider the solar in operating conditions where there are not other fields.

The Kaleidagraph software which is a thoughtfully designed graphing and data analysis application for research scientists permits us to convert complex data obtained during experimentation into transient curves after plotting.

Figure 2 is a sample model of grain under magnetic field.

The calculation of transient voltage decay expression by using boundaries conditions and quasi-neutral base theory gives [7]:

- For linear zone

$$
V(t)=-\frac{V_{T}}{\tau_{c}} \cdot t+V_{T} \cdot \ln \left(q_{0} \cdot \theta_{k, j}(\omega)\right)
$$

This is a linear function with a negative slop $-\frac{1}{\tau_{c}}$.

- For exponential zone

$$
V(t)=V_{T} \cdot q_{0} \cdot \theta_{k, j}(\omega) \cdot \mathrm{e}^{-\frac{t}{\tau_{c}}}
$$

This is a time dependent exponential decay function.

The Equations (3), (4), (5) and (6) are boundaries conditions 


$$
\begin{aligned}
& \left.\frac{\partial \delta(x, y, z, t)}{\partial z}\right|_{z=0}=\frac{S f}{D^{*}} \delta(x, y, z=0, t) \\
& \left.\frac{\partial \delta(x, y, z, t)}{\partial z}\right|_{z=H}=\frac{S b}{D^{*}} \delta(x, y, z=H, t) \\
& \left.\frac{\partial \delta(x, y, z, t)}{\partial x}\right|_{x= \pm a}= \pm \frac{S g x}{D^{*}} \delta(x= \pm a, y, z, t) \\
& \left.\frac{\partial \delta(x, y, z, t)}{\partial y}\right|_{y= \pm b}= \pm \frac{S g y}{D^{*}} \delta(x, y= \pm b, z, t)
\end{aligned}
$$

$S f, S b, S g$ are the recombination velocity of minority charge carriers respectively at surfaces $z=0, z=H$ and $x= \pm a$ (or $y= \pm b$ ). $a, b$ and $H$ are the grain sizes as indicated on Figure 2.

$D^{*}, L^{*}$ are also respectively the electrons diffusion coefficient and length diffusion of charge carriers.

\section{Effective Lifetime Measurement}

Our approach is based on the linear approximation of transient voltage decay because in low injection it permits to avoid impedances effects [8]. The following curve presents the different zone of transient voltage curve.

After identification of linear zone of the curve, we fit this zone and we get a linear regression line indicated on Figure 4.

In Figure 5, the linear regression line equation is found with a good correlation coefficient $R$. The slope of this linear regression line is dependent to the effective lifetime of minority charge carriers.

$$
|\alpha|=\frac{V_{T}}{\tau_{c}}
$$

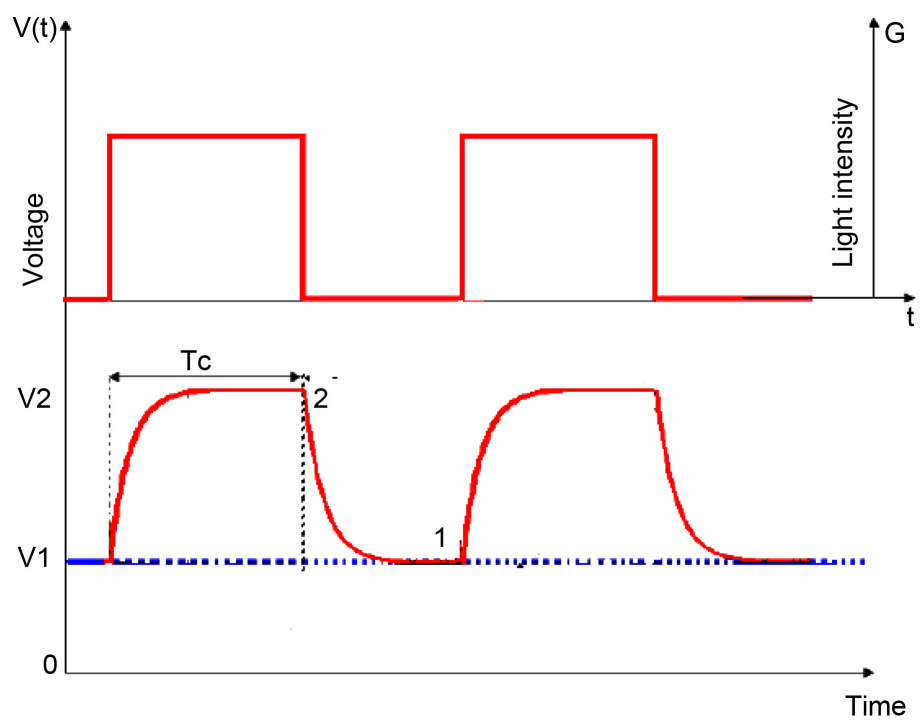

Figure 3. Operating mode. 


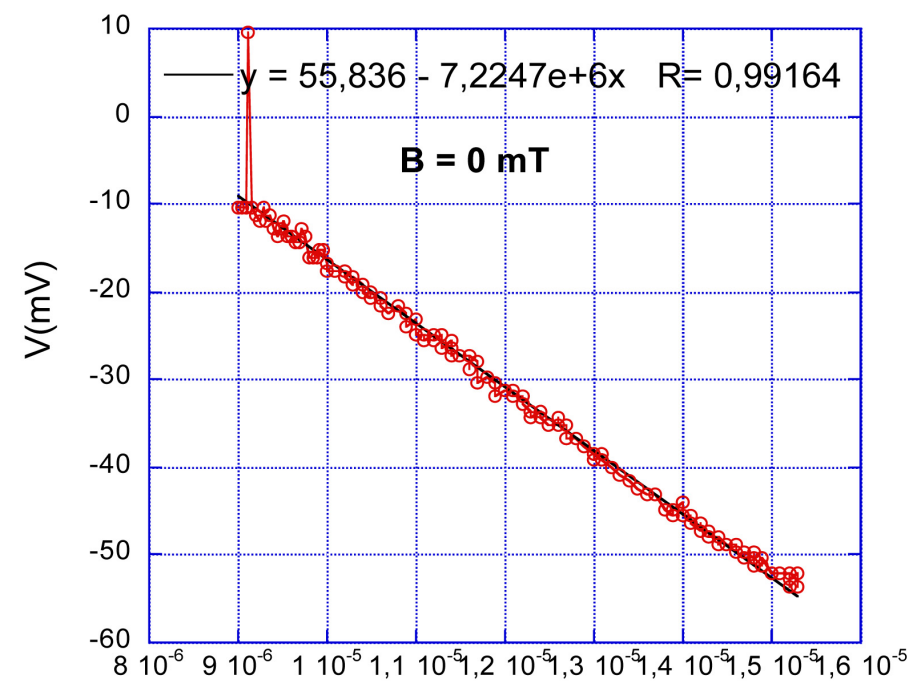

$\mathrm{t}(\mathrm{s})$

Figure 4. A linear regression line with it equation.

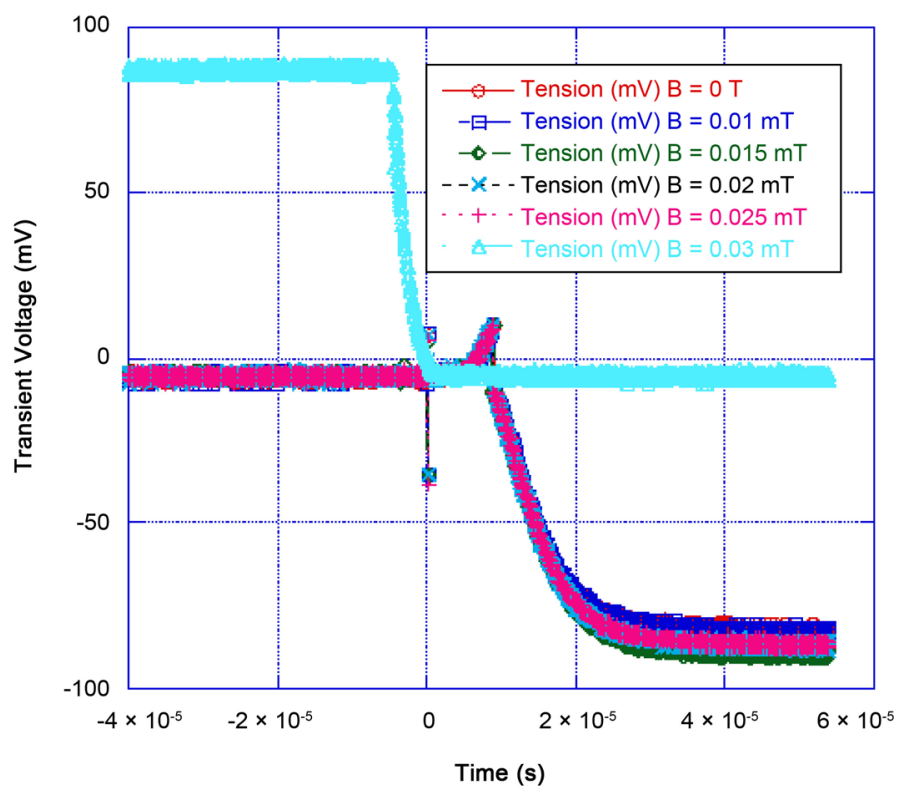

Figure 5. Transient volatage decay curves versus magntic field.

where $\alpha$ is the slope of the transient voltage curve, $V_{T}$ is the thermic voltage and $\tau_{c}$ is the minority charge carrier effective lifetime.

\section{Results and Discussions}

After registration of transient voltage data on digital scope, we use Kaleidagraph software to make simulations. Then, we get the curves of transient voltage decay for various magnetic field according to the time.

By observing the profiles of the curves, we note two types of decay: linear decay and exponential decay. The linear decay is major than exponential decay for all curves because low injection used the permit to avoid impedance effects. The 
Table 1. Effective lifetime values versus magnetic field.

\begin{tabular}{ccccccc}
\hline$B(\mathrm{mT})$ & 0 & 0.01 & 0.015 & 0.02 & 0.025 & 0.03 \\
$\tau_{c}(\mathrm{~s})$ & $23 \times 10^{-6}$ & $23 \times 10^{-6}$ & $21 \times 10^{-6}$ & $20 \times 10^{-6}$ & $19 \times 10^{-6}$ & $6.5 \times 10^{-6}$ \\
\hline
\end{tabular}

transient voltage drop is less sensible for magnetic field values $B \prec 0.03 \mathrm{mT}$ than for magnetic field values $B=0.03 \mathrm{mT}$.

We also remark that for magnetic field values $B=0.03 \mathrm{mT}$ (curve in blue), the decay is very fast at the beginning of transient state to be slow at the end.

After the fitting of all the analyzed curves, we note that the exponential zone is minor than the linear zone. We got ideal types of open circuit transient voltage decay according to Dariwhal and Mahan conditions [1] [9].

The results of curves fittings are noted on the following Table 1.

The results analysis indicates that the charge carriers' effective lifetime decreases with the magnetic field. These results are in agreement with theoretical results obtained and published by Alain et al. and Sam et al. [7] [8]. However, we remark that for magnetic values $B \prec 0.02 \mathrm{mT}$, the variations are slow. The decay becomes sudden when magnetic field values exceed $0.02 \mathrm{mT}$. Indeed, the charge carriers' effective lifetime is reduced of $55 \%$ for magnetic field values variations $0.025 \mathrm{mT} \prec B \prec 0.03 \mathrm{mT}$. This decay explains the fast recombination of charge carriers when the magnetic field increases. The extension of space charge region width with the magnetic field increase [10] explains an important disappearance of charge carriers. Also, the decrease of diffusion length with magnetic field explains these variations of charge carriers' effective lifetime.

\section{Conclusion}

In this paper, we made an experimental study for lifetime measurement under magnetic field in transient dynamic state. The experimental set-up is presented. The technic of lifetime measurement is conducted from the open circuit voltage decay with electrical excitation and presented. The 3D approach permits to get transient voltage expressions respectively in linear zone and exponential zone. The transient voltage curves are plotted with experimental data by the using of Kaleidagraph software. The linear fit gives minority charge carries effective lifetime by using Equation (7) because the linear zone presents an ideal decay for lifetime measurement. The results obtained indicate the decay of minority charge carrier effective lifetime with magnetic field increase. These experimental results are in good agreement with those published in the literature and experimentally. Our study is therefore validated after all these observations.

\section{Conflicts of Interest}

The authors declare no conflicts of interest regarding the publication of this paper.

\section{References}

[1] Dhariwhal, S.R. and Vasu, N.K. (1981) A Generalised Approach to Lifetime Mea- 
surement in p-n Junction Solar Cells. Solid-State Electronics, 24, 915-927. https://doi.org/10.1016/0038-1101(81)90112-X

[2] Gossick, B.R. (1953) Post-Injection Barrier Electromotive Force of $p-n$ Junctions. Physical Review Journals Archive, 91, 1012. https://doi.org/10.1103/PhysRev.91.1012

[3] Lederhander, S.R. and Giacoletto, L.J. (1955) Measurement of Minority Carrier Lifetime and Surface Effects in Junction Devices. Proceedings of the IRE, Vol. 43, 477-483. https://doi.org/10.1109/JRPROC.1955.277857

[4] Green, M.A. (1983) Minority Carrier Lifetime Using Compensated Differential Open Circuit Voltage Decay. Solid-State Electronics, 26, 1117-1122. https://doi.org/10.1016/0038-1101(83)90011-4

[5] Ray, U.C., Agarwal, S.K. and Jain, S.C. (1982) Theory and Experiments on Open Circuit Voltage Decay of p-n Junction Diodes with Arbitrary Base Width, Including, the Effects of Built in Drift Field in the Base and Recombination in the Emitter. Journal of Physic, 53, 9112.

[6] Sam, R., Zouma, B., Zougmoré, F., Koalaga, Z., Zoungrana, M. and Zerbo, I. (2012) $3 \mathrm{D}$ Determination of the Minority Carrier Lifetime and the $p-n$ Junction Recombination Velocity of a Polycrystalline Silicon Solar Cell. IOP Conference Series. Materials Science and Engineering, Ouagadougou, 17-22 October 2011, Vol. 29. https://doi.org/10.1088/1757-899X/29/1/012018

[7] Sam, R., Kaboré, K. and Zougmoré, F. (2016) A Three-Dimensional Transient Study of a Polycritsalline Silicon Solar Cell under Constant Magnetic Field. International Journal of Engineering Research, 5, 93-97.

[8] Diasso, A., Sam, R. and Zougmoré, F. (2020) External Magnetic Field and Air Mass Effects on Carrier's Effective Lifetime of a Bifacial Solar Cell under Transient State. Research Journal of Applied Sciences, Engineering and Technology, 4, 140-146. https://doi.org/10.19026/rjaset.17.6026

[9] Muralidharan, R., Jain, S.C. and Jain, U. (1982) Determination of the Minority Carrier Lifetime in the Base of a Back-Surface Field Solar Cell by Forward Current-Induced Voltage Decay and Photovoltage Decay Methods. Solar Cells, 6, 162-176. https://doi.org/10.1016/0379-6787(82)90064-3

[10] Diasso, A., Sam, R., Yacouba, N.T. and Zougmoré, F. (2020) Effects of External Magnetic Field and Air Mass on Space Charge Width Extension of a Bifacial Solar Cell Front Side Illumination. International Journal of Energy and Power Engineering, 9, 29-34. https://doi.org/10.11648/j.ijepe.20200903.11 